\title{
An Energy-Efficient routing protocol based on TABU-Genetic Strategy in Wireless Sensor Network
}

\author{
Varsha \\ Research Scholar, Computer Science engineering, IKG Punjab Technical University, Kapurthala 144601,(Punjab) \\ India \\ barkhabright@gmail.com \\ ManjuBala \\ Khalsa College of Engineering \& Technology, Amritsar 143001, (Punjab), India. \\ drmanju571@gmail.com \\ Manoj Kumar \\ DAV institute of Engineering, Management \& Technology, Jalandhar 144001 (Punjab), India \\ drmanojkumarindia@gmail.com \\ ABSTRACT-
}

In Swarm Intelligence, various techniques are being planned as far as ACO, PSO, Fish Swarm, Bats Swarm, Bacterial Foraging, TABU, GA search and so forth. TS and GA is a single algorithm that firstly creates a set of random valid solutions, and for several iterations it optimizes them using a TS-based method. Afterwards, it takes this set of optimized solutions as the initial population for the GA, and iterates until the adopted stop criteria have been met. The objective of this research paper is to implement TABU-GA search to make the protocol more efficient and effective. This paper proposed MSEEC (multilevel stable and energy efficient clustering protocol) utilizing TABU-GA mechanism in the territory of $200 \mathrm{~m} \times 200 \mathrm{~m}$. The recreation is done under the MATLAB 2013 a environment and observed the performance of TABU-GA MSEEC against MSEEC protocol on $4 \%$ increase in the case of first node dead (FND) and $28 \%$ increase in the case of last node dead (LND).

Keywords-Wireless sensor network, heterogeneity, TABU-GA mechanism, MATLAB, FND, LND.

Date of Submission: May 18, 2019

Date of Acceptance: June 05, 2019

\section{Introduction}

The Sensors are mainly used to sense the environment and to gather the data to a centralized location. Advent of processing devices and networks makes it as "Wireless Sensor Network". Development in Semi-conductor technology and Networking methods has stimulated the use of sensor networks for observing and information collection. In Wireless sensor network, information collected by sensors is gathered at a distant location for analyzing and computation purpose via wireless links. Some applications of wireless sensor network include medical, environmental, transportation, military, entertainment, homeland, defense, and crisis management etc. Alike to other communication systems, wireless sensor network systems development has a diversity of origins. The history of development can be briefly alienated into four phases: [1]

Phase 1: During the cold war period, there was a need to monitor and detect the positions of enemies which gave birth to number of projects such as Sound Surveillance System and radar networks developed by United States.

Phase 2: DARPA (Defense Advanced Research Projects Agency) of United States Department of Defense initiated the research programs in the early 1980s that were basically focused on advance developments on new technologies and protocols of wireless sensor networks.

Phase 3: Projects undertaken by DARPA laid the foundation for military applications developments based on wireless sensor networks. Huge amount of money spent on newer technologies made the development faster in early 1990s.

Phase 4: Recent advancements in semi-conductor technologies and networking techniques directed an innovative stage in the growth of sensor network technology. In 2000's IEEE released the first version of IEEE Standard i.e. 802.15.4 standard "Low Rate Wireless Personal Area Networks" which is the base for recently introduced standards such ZIGBEE.

Wireless sensor network contains battery functioned small nodes which are positioned over a wide geographical area to monitor the events and to accumulate the collected data to a distant centralized location called as base station. odes are installed in such a way that the entire area is in the coverage of wireless nodes the deployed nodes sense the data from its neighborhood and transmit the collected data for further processing [2]. The main distinction between ad hoc networks and Wireless Sensor Networks is their applications area. Ad-hoc networks primarily focus on communications aspects whereas wireless area 
networks focus more on monitoring and information collection [3].Wireless nodes are bound by several resource restrictions such as the memory availability, battery power, bandwidth requirement and the data rate. These tiny nodes may work for a longer duration of time from few months to many years depending upon the application requirements, so the battery power needs to be employed proficiently so as to extend the network life time. Sleep Mode operation of nodes is efficient way to increase the life time. Nodes wakes-up only when there is a need to sense the environment. In order to save the maximum battery life, the sensor node should communicate by using a special routing technique. Heterogeneous Wireless sensor network is especially valuable in genuine arrangements since they are all the more near genuine circumstances. Routing is a great challenge testing issue in the structure of WSNs. The stable and energy efficient clustering protocol (SEEC) have two highlights like it is energy proficiency situated convention and stability oriented protocol. In SEEC convention the entire system is partitioned into several different clusters. Every cluster has strong advance nodes $(\mathrm{AN})$ and $(\mathrm{NN})$ normal nodes are conveyed arbitrarily in the system .The normal nodes go about as detecting specialists and advance nodes collect information and send it to the sink. Then again, multilevel stable and energy efficient clustering protocol is utilized which is an expansion over the SEEC convention. In this convention, normal nodes $(\mathrm{NN})$, advance nodes (AN) and super nodes (SN) are utilized dependent upon their underlying energy. Advance nodes having $\alpha$ time more energy and super nodes acquiring $\beta$ times more energy in comprise of normal nodes. The individuals from cluster will send information to advance and super head. After getting the information, the aggregation operation will be started which is reducing the duplicity of the information in the network. This convention is an upgrade over SEEC convention since it has progressively unstable duration. Since it is energy proficient convention however it experiences the heterogeneity of a protocol. If the Super Node(SN) which go about as a Cluster Head(CH) is die, the entire Network will goes out and Normal Node will coordinate Communicate to Advanced Node(AN) and energy Consumption is so high which will lessen the Network Lifetime of a Network. The Whole Network is Static and it additionally Causes Flooding at sink.

The Contribution to this paper is to enhance the MSEEC using TABU and GA search mechanism which is developed for solving optimization problems for higher level heuristic procedure. It has the ability to find out the optimal and near optimal solution to problems. This paper addresses the matter of routing in WSNs using TABU and GA search with the aim of maximize the network lifetime. Under contribution this paper proposes the TABU and GA multilevel stable and energy efficient clustering protocol (TABU-GA MSEEC) for the purpose of routing in WSN with the aim of maximize the network lifetime in the area of $200 \mathrm{~m} \times 200 \mathrm{~m}$.
Remaining paper is in following order: Section 2 elaborates the contribution. Section 3 defines the Proposed protocol. Section 4 depicts the TABU search algorithm and section 5 specifies the hybrid algorithm and Section 6 describes the experimental setup and Section 7 defines the simulation Results and discussion and at last Section 8 formulate with conclusion and future scope.

\section{Contributions}

Following objectives have been considered for the advancement of multilevel stable and energy efficient clustering protocol:

- To enhance multilevel stable and energy efficient clustering protocol (MSEEC) over energy utilization by formulating significant criteria for routing.

- For uniform energy utilization over the system, another strategy for information transmission over the system is proposed.

\section{Proposed protocol}

This paper described TABU-GA MSEEC protocol for WSNs in the presence of node heterogeneity with the routing done by TABU-GA search against the area of $200 \mathrm{~m} \times 200 \mathrm{~m}$ using TABU-GA search for MSEEC energy efficient clustering protocol. In TABU-GA MSEEC, there are three types of nodes; normal node, advance node and super node based on their initial energy value. The eight advance nodes and eight super nodes are placed while rest of the normal nodes are placed. $\mathrm{M}_{\mathrm{sn}}$ is the proportion of entire number of nodes having $\alpha$ times greater energy in comprise of normal nodes called super nodes. The remaining nodes having energy denotes by $\mathrm{E}_{0}$.

$\mathrm{P}=\mathrm{p} 1+\mathrm{p} 2$ where $\mathrm{p} 1$ is the entire number of normal nodes attached to advance nodes and $\mathrm{p} 2$ is the entire normal nodes attached to super nodes. The entire energy equation of three level nodes is given below:-

$E_{\text {total }}=$ p. $E_{0}+M_{A N} \cdot(1+\alpha) \cdot E_{0}+M_{s n} \cdot(1+\beta) \cdot E_{0}$

Therefore, the three-level M-SEEC has $\left(\alpha \cdot \mathrm{M}_{\mathrm{AN}}+\beta \cdot \mathrm{M}_{\mathrm{SN}}\right)$ times more energy. From the figure 1, we can easily see the randomization of normal nodes. 


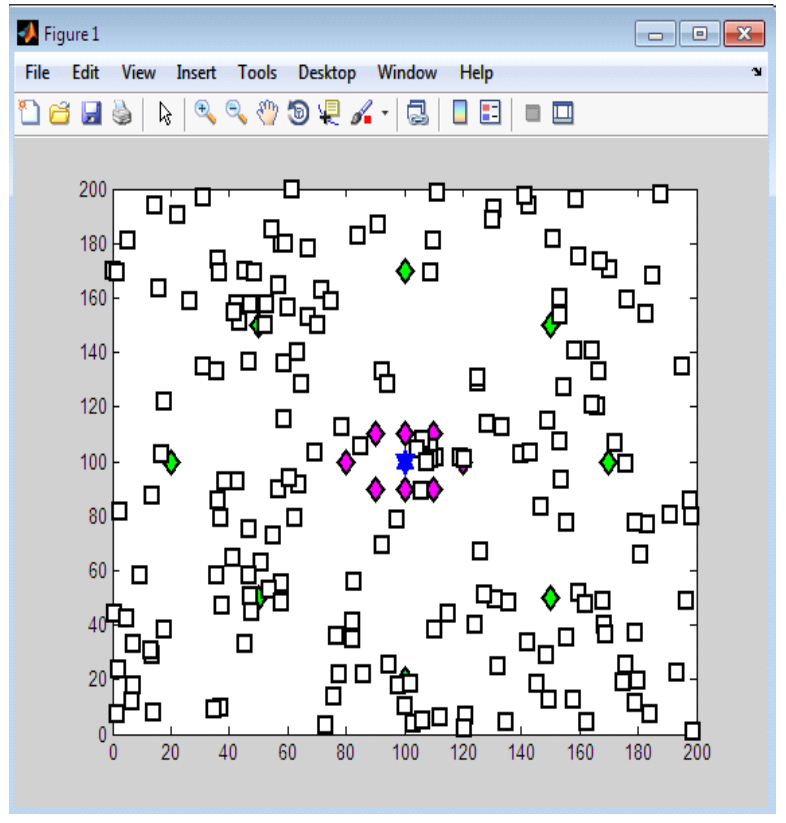

Figure 1.Snapshot of the entire network when 200 nodes are distributed in $200 \mathrm{~m} \times 200 \mathrm{~m}$ network.

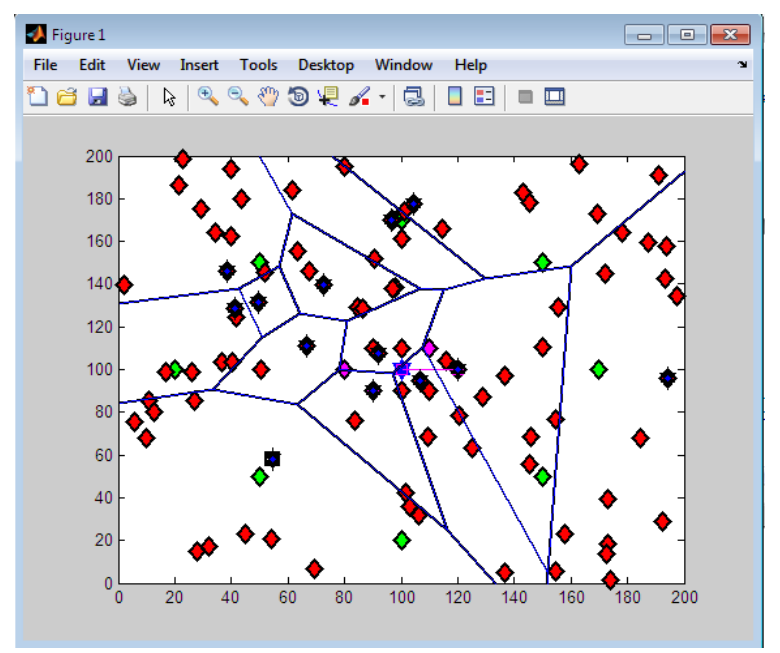

Figure 2: Selection of cluster heads

This Figure represents the scenario of cluster heads, how the clustering is done by using the suitable clustering algorithm. Red diamond shape nodes represents the dead nodes and blue dotted line represents the data communication.

The following parameters are considered:

$\mathbf{n} 1$ is the number of NNs,

mAN is the number of ANs.

Then the total nodes in the network are $\mathbf{N}=(\mathbf{n} 1+\mathbf{m A N})$.

$\mathbf{n A}$ is the number of NNs in each cluster

(wherenA =n1 /mAN)[10]

Each AN is located at a position of distance $\mathbf{R} 1$ and angle $\boldsymbol{\theta}$ with respect to the BS,

that is, for each $A N \mathbf{j}$ there is $(\mathbf{R} \mathbf{1}, \boldsymbol{\theta} \mathbf{j})$ where $\mathbf{j}=\mathbf{1}, \mathbf{2}, \ldots, \mathbf{m A N}$.

The distance R1 is the same for all ANs, but the angle $\theta$ is changed.
The $\theta \mathbf{j}$ is calculated as: $\boldsymbol{\theta} \mathbf{j}=\boldsymbol{\varnothing}+(\mathbf{j}-\mathbf{1}) \times \mathbf{3 6 0} / \mathbf{m A N}$ where $(\mathbf{j}=\mathbf{1}, \mathbf{2}$, ..., mAN )

\section{$\emptyset=180 / \mathrm{mAN}[9]$}

in each cluster $\mathbf{j}$, there are $\mathrm{NN} \mathbf{i}$ located at $(\mathbf{r} \mathbf{i}, \boldsymbol{\gamma} \mathbf{i}) \mathbf{j}$ with respect to the BS,

wherei $=\mathbf{1}, \mathbf{2}, \ldots, \mathbf{n A}$; such that, $\mathbf{r} \mathbf{i}>=\mathbf{R} 1$ and $(\boldsymbol{\theta} \mathbf{j}-\boldsymbol{\emptyset})<\gamma \mathbf{i}<(\boldsymbol{\theta} \mathbf{j}$ $+\varnothing)[9]$

The NNs are uniformly distributed over the sensor field AN has $\boldsymbol{\alpha}$ times more energy than NNs.

\section{TABU Search Algorithm}

Essentially, the TABU search was generated by Fred Glover and this instrument is enlivened by human memory with the end goal to enhance the productivity of the investigation procedure, one needs to store the neighborhood data (like the present estimation of the goal work) yet in addition of some data identified with the investigation procedure. To locate the ideal arrangement, the TABU search produces the underlying arrangement attainably. The underlying arrangement turns out to be at the same time the best arrangement and current arrangement and by and large this arrangement is embedded into the memory list, called TABU list. This list is utilized to keep the cycling. On the off chance that TABU list is full; the most established component of TABU list is evacuated and new is included. The TABU scan acquired the area answers for the present arrangement, and updates the present arrangement with TABU list. In each time, the arrangement of neighbors of current arrangement is make by the area tasks and the neighbor with the most noteworthy esteem is check as the new best arrangement in TABU list and new best arrangement is acknowledged for the choice of hopeful arrangement, generally the arrangement having the following most astounding quality will turns into the competitor arrangement. Presently, the expense of new best arrangement is contrasted and the best arrangement generally cycles is increments and new best arrangement is updated into the TABU list and mentioned as the present answer for the next arrangement. The means of TABU search are characterized underneath:-

It could be communicated as seeks after ( $i^{*}$ is the best arrangement found up until now and $\mathrm{k}$ the cycle counter):

Stage 1. Pick the first solution I in S. Set $i^{*}=i$ and $k=0$.

Stage 2. Set $\mathrm{k}=\mathrm{k}+1$ and create a subset $\mathrm{V}^{*}$ of arrangement in $\mathrm{N}(\mathrm{i}, \mathrm{k})$

Stage 3. Pick a best $\mathrm{j}$ in $\mathrm{V}^{*}$ (regarding for to some altered capacity $\mathrm{f} \sim$ ) and set $\mathrm{i}=\mathrm{j}$.

Stage 4. In the event that $\mathrm{f}(\mathrm{i})<\mathrm{f}\left(\mathrm{i}^{*}\right)$ set $\mathrm{i}^{*}=\mathrm{i}$.

Stage 5 . In the event that a ceasing condition is met, stop. Else go to Step $2[4,5,6]$. 


\section{The TABU-GA MSEEC protocol}

The TABU-GA MSEEC algorithm implemented was based on the TS-GA hybrid model proposed by Zdansk\&Pozivil in 2002. This model is intended to combine the TS and GA in a single algorithm that firstly creates a set of random valid solutions, and for several iterations it optimizes them using a TS-based method. Afterwards, it takes this set of optimized solutions as the initial population for the GA, and iterates until the adopted stop criteria have been met. For the implemented hybrid agent, the TS output is not written in the main memory, as the TS pure agent, but it is stored in a transition memory, which has an interface with the module that implements the GA. After the stop criteria for the TS have been met, the GA module uses the solutions in this memory as initial population and performs iterations until the stop criteria have been met. At last, the best solution produced by the GA is written to the main memory [7-10].

Methodology has shown various steps required to successfully simulate the proposed work. Subsequent are the various steps with brief detail:

Step 1: Start:-First of all, process is initialized.

Step2: Deploy WSNs: - After initializing, the Wireless sensor networks are deployed.

Step3:Apply Radio energy dissipation model to evaluate CHs :After applying the Radio energy dissipation model, Radio energy dissipation model is applied to it for the evaluation of cluster heads.

Step4: Associate member nodes with CHs:-Then the member nodes are associated with the cluster head evaluated in Step 4.[11,12]

Step5: Apply Tabu-GA search algorithm to find path between CHs and base Station:-After that, Tabu search algorithm is applied to find path between cluster heads and the nodes.

Step6: Transmit data:-Then transmission of the data will be done.

Step7: Evaluate Energy dissipation: - After transmission of the data, dissipation of the energy is evaluated and after updating the remaining energy, dead nodes will be evaluated.

Step8: To find node dead:-Then it is found that whether the node is dead or not. If the node is dead, then goto step 10 , otherwise goto step 3 .

Step9: Count dead:-Now count the total dead nodes, a node is assumed to be dead if it has no more energy to transmit of receive data.

Step10: To find dead equal to $\mathrm{n}$ :-In this step, it is found that whether the nodes are equal to $\mathrm{n}$ or not. If they are equal, then goto step 12, otherwise goto step 3 .

Step11: Network lifetime:-Here the network lifetime will be evaluated.

\section{Step12: End}

\section{Experimental Setup}

The MATLAB simulator is used for the implementation. In the first case, 200 nodes are placed in which 8 advanced nodes and 8 super nodes are fixed and rest of the normal nodes are placed inside the area of $200 \times 200 \mathrm{~m}^{2}$. Here, the parameters are used in the simulation are mentioned below in table 1 .

Table 1:Network Parameters

\begin{tabular}{|l|l|}
\hline \multicolumn{1}{|c|}{ Parameter } & Value \\
\hline Terrain of the network & $200 \times 200$ \\
\hline Position of Sink & 100,100 \\
\hline Total number of Nodes & 200 \\
\hline $\begin{array}{l}\text { Probability(p) to be a } \\
\text { cluster head }\end{array}$ & 0.1 \\
\hline Startup Energy(Eo) & $0.5 \mathrm{~J}$ \\
\hline Transmiter_energy & $50 \mathrm{~nJ} / \mathrm{bit}$ \\
\hline Receiver_energy & $50 \mathrm{~nJ} / \mathrm{bit}$ \\
\hline Free space & $10 \mathrm{nj} / \mathrm{bit} / \mathrm{m}^{2}$ \\
\hline Multipath & $0.0013 \mathrm{pJ} / \mathrm{bit} / \mathrm{m}^{4}$ \\
\hline
\end{tabular}

\section{Simulation Results and Discussion}

On applying TABU-GA search mechanism, the following results will come out. For simulation MATLAB 2013 a tool is used for easy computing of results. All results were compared with the basic MSEEC and TABU-MSEEC and then TABU-GA MSEEC protocol. The performance metrics used in the results shown that the proposed TABU-GA MSEEC gives the larger stability period, more efficient in favor of energy and high network lifetime than the other protocols. The stability period of TABU-GA MSEEC is much longer than that of other cases. This paper observed that TABU-GA MSEEC take some advantage in forms of death of first node and last node, which enhances the network stability.

\section{Number of dead nodes}

Figure 2depicts that in MSEEC network the first and last sensor node death is after 5000and 10000 rounds. However, for TABU MSEEC network the first node and last node dead is after 6500 and 15000 rounds respectively and from TABU-GA MSEEC, the first node and last node dead is after 6800 and 14000 rounds From the results, it is clearly shown that the performance of TABU-GA MSEEC is much more in case of first node dead (FND) and also more in case of last node dead (LND). Certainly, this change in the performance is due to the modification in the simulation setup because the protocol has the TABU-GA 
based routing. Table 2 calculates the exact reading of the network.

Table 2: First node dead and Last node dead comparison among protocols

\begin{tabular}{|c|l|l|l|}
\hline Dead Nodes & Protocol & FND & LND \\
\hline $\begin{array}{c}\text { All nodes } \\
\text { including } \\
\text { NN, AN and } \\
\text { SN }\end{array}$ & MSEEC & 5000 & 10,000 \\
\cline { 2 - 4 } & $\begin{array}{l}\text { TABU } \\
\text { MSEEC }\end{array}$ & 6500 & 15000 \\
\cline { 2 - 4 } & $\begin{array}{l}\text { TABU-GA } \\
\text { MSEEC }\end{array}$ & 6800 & 14000 \\
\hline
\end{tabular}

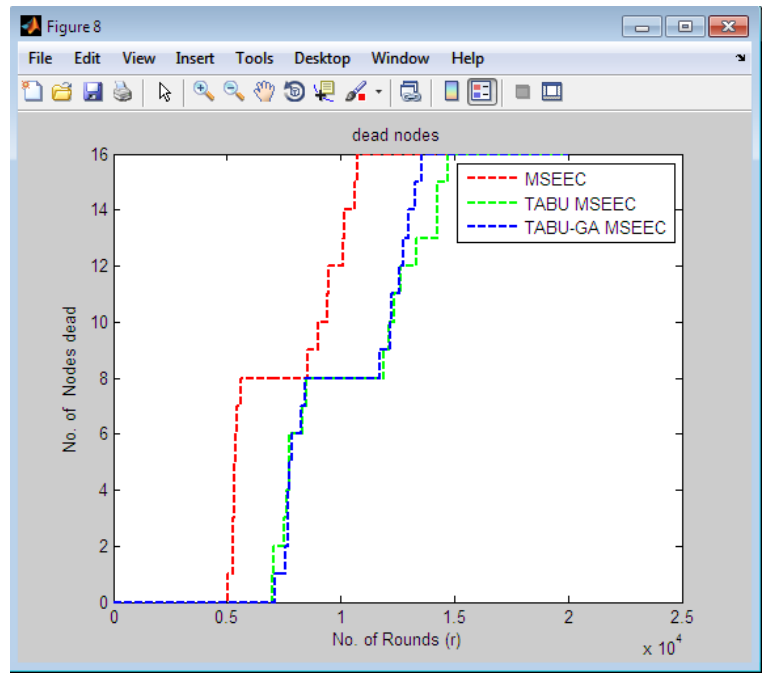

Figure 2. Number of Packet transferred vs. no of rounds for 200 nodes in $200 \times 200$ network.

\section{Number of Packet send to base station}

Figure 3 represents the number of packet transferred against the number of rounds. From the figure, it is clearly shown that the red dotted line represents the number of packet transferred in case of MSEEC protocol and blue dotted line represents the number of packet transferred in case of TABU-MSEEC and green dotted line represents the number of packet transferred in case of TABU-GA MSEEC protocol. To evaluating the network lifetime of a network, the number of packet transferred was calculated. From the simulation, it is observed that at the round of 15000 , thepacket transferred are 188 in case of MSEEC protocol and 192 in case of TABU MSEEC and 193 in case of TABU-GA MSEEC protocol. Proposed protocol has improvement which shows that TABU-GA MSEEC performs better than that of MSEEC and TABU-MSEEC protocol.Table 4 calculates the exact reading of the network.
Table 3: Readings of packet send to base station with respect to rounds among different protocols

\begin{tabular}{|l|l|l|}
\hline $\begin{array}{l}\text { Packet send to } \\
\text { base station }\end{array}$ & Protocol & No of rounds \\
\hline $\begin{array}{l}\text { All nodes } \\
\text { including NN, }\end{array}$ & MSEEC & 188 \\
\cline { 2 - 3 } AN and SN & TABU MSEEC & 192 \\
\cline { 2 - 3 } & $\begin{array}{l}\text { TABU-GA } \\
\text { MSEEC }\end{array}$ & 193 \\
\hline
\end{tabular}

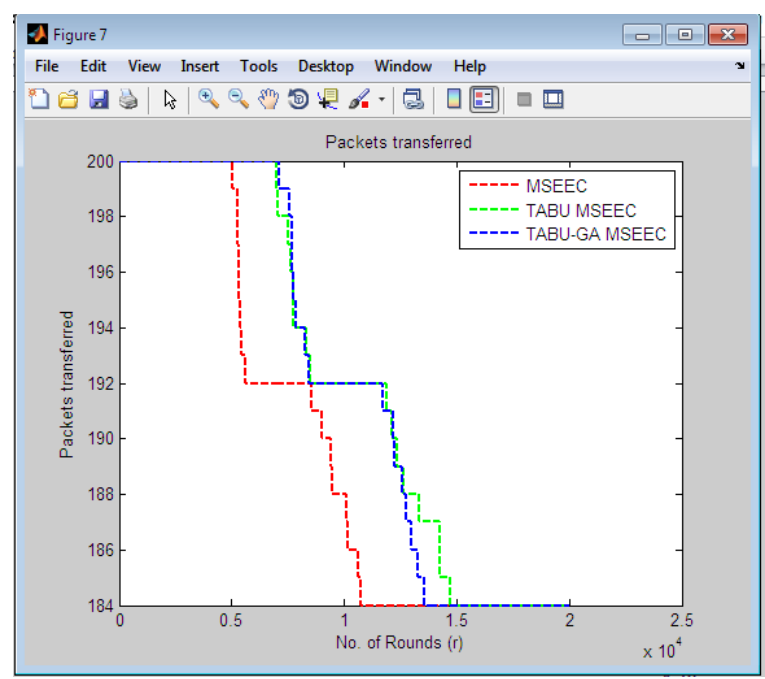

Figure 3.Packet send to base station vs. no of rounds for 200 nodes in $200 \times 200$ network.

\section{Conclusion And Future Scope}

In this paper, MSEEC,TABU MSEEC and TABU-GA MSEEC protocol has been proposed for wireless sensor network. Here, the performance comparison is done and found that TABU-GA MSEEC is $4 \%, 26 \%$ raise in case of first node dead (FND)with MSEEC and TABU-MSEEC protocol and $28 \%$ raise in case of last node dead (LND) in MSEEC protocol. Here, the observations reveal that the performance of TABU-GA SEARCH is very high as compared to the MSEEC protocol because TABU-GA search chooses the most effective solution. As a future work, gathered data can be utilized to decide the impact of the path loss in applications that relies upon RSSI and path loss exponent, for example, RSS-based confinement and energy analysis and also work on clustering as well as routing with other bio-inspired algorithms like cuckoo search, flower pollination, spider monkey optimization, Glow-worm, Meer cat Clan algorithm.

\section{References}

[1] Farouk, F., Rizk, R., \&Zaki, F. W. (2014). Multi-level stable and energy-efficient clustering protocol in heterogeneous wireless sensor networks. IET Wireless Sensor Systems, 4(4), 159-169. 
[2] Wang, X., Qian, L., Wu, J., \& Liu, T. (2010). An energy and distance based clustering protocol for wireless sensor networks. In Novel Algorithms and Techniques in Telecommunications and Networking (pp. 409412).Springer, Dordrecht.

[3] Li, X., Li, N., Chen, L., Shen, Y., Wang, Z., \& Zhu, Z. (2010, March). An improved LEACH for clustering protocols in wireless sensor networks. In 2010 International Conference on Measuring Technology and Mechatronics Automation (Vol. 1, pp. 496-499).IEEE.

[4] Orojloo, H., \&Haghighat, A. T. (2016). A Tabu search based routing algorithm for wireless sensor networks. Wireless Networks, 22(5), 1711-1724.

[5] Messaoud, R. B., \&Ghamri-Doudane, Y. (2015, September). QoI and energy-aware mobile sensing scheme: A tabu-search approach. In 2015 IEEE 82nd Vehicular Technology Conference (VTC2015-Fall) (pp. 16).IEEE.

[6] Amuthan, A., \&Thilak, K. D. (2016, October). Survey on Tabu search meta-heuristic optimization.In 2016 International Conference on Signal Processing, Communication, Power and Embedded System (SCOPES) (pp. 1539-1543).IEEE.

[7] Habib, S. J., \&Marimuthu, P. N. (2017, April). Reputation analysis of sensors' trust within tabu search.In World Conference on Information Systems and Technologies (pp. 343-352).Springer, Cham.

[8] Kuo, S. Y., \& Chou, Y. H. (2017). Entanglementenhanced quantum-inspired tabu search algorithm for function optimization. IEEE Access, 5, 13236-13252.

[9] Vijayalakshmi, K., \&Anandan, P. (2018). A multi objective Tabu particle swarm optimization for effective cluster head selection in WSN. Cluster Computing, 1-8.

[10]Qu, W., \& Yang, M. (2014, June). An energy-efficient routing control strategy based on genetic optimization.In Proceeding of the 11th World Congress on Intelligent Control and Automation (pp. 2038-2041).IEEE.

[11]Ganesh, S., \&Amutha, R. (2010). Real Time and Energy Efficient Transport Protocol for Wireless Sensor Networks.International Journal of Advanced Networking and Applications. arXiv preprint arXiv:1006.2691.

[12]Basavaraj, G. N., \&Jaidhar, C. D. (2019). Intersecting Sensor Range Cluster-based Routing Algorithm for Enhancing Energy in WSN. International Journal of Advanced Networking and Applications, 10(4), 39383943. 